\title{
MODERN DEVELOPMENTS IN THE GEOMETRY OF NUMBERS ${ }^{1}$
}

HANS ZASSENHAUS

Modern geometry of numbers is chiefly concerned with two problems, the one is a packing problem, the other a covering problem. In both cases, an estimate of the density of a subset of some space is required.

The density notion of which I am speaking has its roots in the atomic theory of matter. It may be axiomatized as follows. Let $\partial$ be a non-negative real valued set function (possibly $\infty$ ) on a space $E$ satisfying the conditions

$$
\begin{array}{cr}
\partial(\varnothing)=0 & (\varnothing \text { the empty subset }), \\
\partial(A \cup B) \geqq \partial(A)+\partial(B) & \text { if } A \cap B=\varnothing .
\end{array}
$$

Furthermore assume that there is a permutation group $G$ (symmetry group) of $E$ given. We say that the subset $A$ of $E$ has density $\partial(A)$ if

$$
\begin{array}{rrr}
\partial(\alpha A) & =\partial(A) & (\alpha \in G), \\
\partial(\alpha A \cup B) & =\partial(\alpha A)+\partial(B) & \text { if } \alpha A \cap B=\varnothing ;(\alpha \in G) .
\end{array}
$$

Thus any symmetry applied to a point set with a density yields a point set of the same density. The union of two disjoint sets both of which have a density has itself a density and the density of the union is equal to the sum of the densities of the summands. For example, let $E=E_{n}$ be the $n$-dimensional Euclidean space. Let $G$ be the group of the isometries (rigid movements) of the $E_{n}$. For any point set $S$, denote by $n(t, S)$ the minimum number of points of $S$ belonging to a hypercube:

$$
a_{i} \leqq x_{i}<a_{i}+t \quad(i=1,2, \cdots, n)
$$

Then

$$
\partial(S)=\lim _{t \rightarrow \infty} \frac{n(t, S)}{t^{n}}
$$

exists, possibly with $\partial(S)=\infty$ and the properties (1), (2) can be verified.

\footnotetext{
${ }^{1}$ An address delivered before the Stanford meeting of the Society on April 22, 1961, by invitation of the Committee to Select Hour Speakers at Far Western Sectional Meetings; received by the editors May 3, 1961.
} 
It turns out that any $n$-dimensional lattice

$$
\begin{array}{r}
L=\left\{P=\left(x_{1}, x_{2}, \cdots, x_{n}\right) \mid x_{i}=a_{i}+\sum_{k=1}^{n} a_{i k} t_{k}(i=1,2, \cdots, n),\right. \\
\left.t_{1}, t_{2}, \cdots, t_{n} \text { rational integers, and } \operatorname{det}\left(a_{i k}\right) \neq 0\right\}
\end{array}
$$

has a density, namely the inverse of the mesh

$$
\Delta(S)=\left|\operatorname{det}\left(a_{i k}\right)\right| \text {. }
$$

Any finite point set has density 0 . Any point set of positive density contains a discrete subset of the same density.

The packing problem consists in estimating from above the density of any point set $S$ satisfying the condition

$P Q \notin X$

$$
(P, Q \in S ; P \neq Q)
$$

for a certain subset $X$ of the $n$-vector space. Thus the competition is between the $X$-admissible point sets of the $E_{n}$ as defined above by condition (A).

The covering problem consists in estimating from below the density of any point set $S$ meeting the condition that for any point $Q$ of the $E_{n}$ there is a point $P$ of $S$ such that

$$
P Q \in X
$$

for the same subset $X$ of the $n$-vector space.

As set $X$ one usually takes a star characterized by the conditions

(a) $X$ is closed.

(b) If $x$ belongs to $X$ and if $0 \leqq \alpha \leqq 1$ then also $\alpha x$ belongs to $X$.

(c) If $x$ is any nonvanishing $n$-vector then either all products by a positive scalar and $x$ belong to $X$ (asymptotic directions) or there is a positive number $f(x)$ such that $x / f(x)$ belongs to $X$ but $(1+\epsilon) x / f(x)$ does not belong to $X$ for any positive number $\epsilon$.

Setting $f(x)=0$ for the zero vector and for asymptotic directions, a continuous real valued function is associated with a star that is characterized by the properties:

$$
\begin{aligned}
f(\alpha x) & =\alpha f(x) \\
f(x) & \geqq 0, \\
f(x) & \leqq 1 \text { if and only if } x \text { belongs to } X .
\end{aligned}
$$

Conversely, any real valued continuous function $f$ satisfying (5), (6) defines an associated star $X$ according to (7). The corresponding star distance 


$$
d(P, Q)=f(P Q)
$$

is characterized by the properties

$$
\text { (homogeneity): } \begin{aligned}
d(P, R) & =\alpha d(P, Q) \text { if } P R=\alpha P Q, \quad \alpha>0, \\
d(P, Q) & \geqq 0
\end{aligned}
$$

reflecting (5), (6).

The best studied examples are provided by the Minkowski distances which are defined simply as star distances that satisfy Fréchet's conditions

$$
\begin{aligned}
& d(P, Q)=d(Q, P) \Leftrightarrow f(x)=f(-x), \\
& d(P, R) \leqq d(P, Q)+d(Q, R) \Leftrightarrow f(x+y) \leqq f(x)+f(y) .
\end{aligned}
$$

The corresponding star is characterized as a centrally symmetric convex closed set of $n$-vectors with the zero vector in the interior.

Examples of symmetric star distances are the norm distances

$$
\begin{aligned}
N_{r}\left(\left(x_{1}, x_{2}, \cdots, x_{n}\right)\right)=\left|\left[\prod_{i=1}^{r} x_{i} \cdot \prod_{j=1}^{c}\left(x_{r+j}^{2}+x_{r+c+j}^{2}\right)\right]^{1 / n}\right| & (r+2 c=n)
\end{aligned}
$$

that are equal to the absolute value of the $n$th root of the norm of any algebraic number $\alpha$ belonging to an algebraic numberfield $E$ of degree $n$ over the rational field such that $x_{1}, x_{2}, \cdots, x_{r}$ are the real algebraic conjugates and, moreover,

$$
y_{r+1} \pm(-1)^{1 / 2} x_{r+c+1}, x_{r+2} \pm(-1)^{1 / 2} x_{r+c+2}, \cdots, x_{r+c} \pm(-1)^{1 / 2} x_{n}
$$

are the $2 c$ complex algebraic conjugates of $\alpha$ over the rational field. Only in the cases: $n=1$ ( $E$ the rational field); $n=2, r=0$ ( $E$ imaginary quadratic) we obtain Minkowski distances. In any event the points associated with the algebraic integers of $E$ form an admissible $n$-dimensional geometric lattice. The square of its mesh multiplied by $4^{-c}$ is equal to the absolute value of the discriminant of $E$.

Classical geometry of numbers as conceived by Minkowski [8; 9], and diligently pursued by Siegel [16], Hlawka [4], Mahler $[6 ; 6 a]$, Mordell [10;11], Davenport [3], Bambah [1], C. A. Rogers [14; 15] and other mathematicians is restricting the competition to $n$-dimensional geometric lattices. The packing problem consists in finding a good estimate of the least upper bound $\Gamma^{*}(X)$ of the densities of the lattices $S$ meeting condition (A). A subsidiary problem is the question whether there is an $X$-admissible $n$-dimensional lattice of density $\Gamma^{*}(X)$. Such a lattice is called $X$-critical or, in the event of stars with star distance $d$, also $d$-critical. The conditions of the existence of $X$ - 
critical lattices have been investigated by $\mathrm{K}$. Mahler [6; 6a]. They certainly exist for convex stars.

Whereas classical geometry of numbers restricts the competition to the admissible $n$-dimensional lattices, statistical geometry of numbers removes it in order to face the underlying geometric problem in a clear and pure way.

The packing problem consists in finding a good estimate of the least upper bound $\Gamma^{* *}(X)$ of the densities of any point set $S$ whatever satisfying condition (A). The covering problem consists in estimating the greatest lower bound $\Gamma_{* *}$ of the density of any point set $S$ satisfying condition (B).

The following generalizations are possible:

(I) Instead of the translation group of a Euclidean space, any continuous transitive transformation group of a topological manifold may form the basic group.

(II) For certain applied problems (packing of sand grains, structure of solid matter), a finite set of $n$-vector sets, say $x_{i k}(1 \leqq i, k \leqq f)$ is given. The competition is between admissible systems of pointsets $S_{1}, S_{2}, \cdots, S_{f}$ of the $E_{n}$. It is required that the densities of these pointsets stand in fixed proportion: $\partial\left(S_{i}\right)=\alpha_{i} \partial\left(\alpha_{i}>0 ; i=1,2, \cdots, f\right)$. The condition of admissibility for the packing problem consists in

$$
P P^{\prime} \notin X_{i k} \quad \text { if } P \in S_{i}, P^{\prime} \in S_{k} .
$$

For the covering problem the condition of admissibility consists in the existence of a point $P$ of some $S_{i}$ for each point $Q$ of the $E_{n}$ such that

$$
P Q \in X_{i}
$$

Early studies in statistical number geometry have been made in connection with the problem of finding the densest packing of solid spheres of fixed diameter in 2 and 3 dimensions. Intuition, observation and experiment point to the conjecture that $\Gamma^{*}=\Gamma^{* *}$. For two dimensions this conjecture has been verified by Thue [18]. ${ }^{2}$ But for 3 dimensions the problem is still an open question. A critical lattice for the packing problem of solid spheres of diameter 1 up to $n=3$ dimensions is the hexagonal lattice which is characterized by the existence of a vector basis $v_{1}, v_{2}, \cdots, v_{n}$ satisfying the conditions: $v_{i} \cdot v_{k}=\left(1+\delta_{i k}\right) / 2$.

${ }^{2} \mathrm{~N}$. Oler [12] pointed out the noncompactness of the metrical space formed by the Jordan polygons used by Thue and rectified the variation method of Thue in a far more general setting [12]. 
C. A. Rogers [15] and L. Fejes Tóth [4a] proved the equality $\Gamma^{*}=\Gamma^{* *}$ for all Minkowski-distances in the plane. The study of the applications to mineralogy and to metallurgy strongly suggests the conjecture that admissible point sets of the $E_{2}$ of maximal density with respect to a given Minkowski-distance $d$ must show some regularity as is the case in the critical lattices. But no precise meaning can be given to this conjecture as long as only infinite admissible pointsets are considered because one may always move about or eliminate in irregular fashion finite subsets without change of density. But finite admissible pointsets always have density zero. We must associate a new number with them by which we quantitatively describe the tightness of the given finite point distribution.

The impression of varying degrees of tightness of finite plane pointsets is caused by 3 factors: area covered, circumference spanned, number of points participating. The following 3 pairs of diagrams illustrate the difference caused by the variation of one at a time only:

(I) Area covered
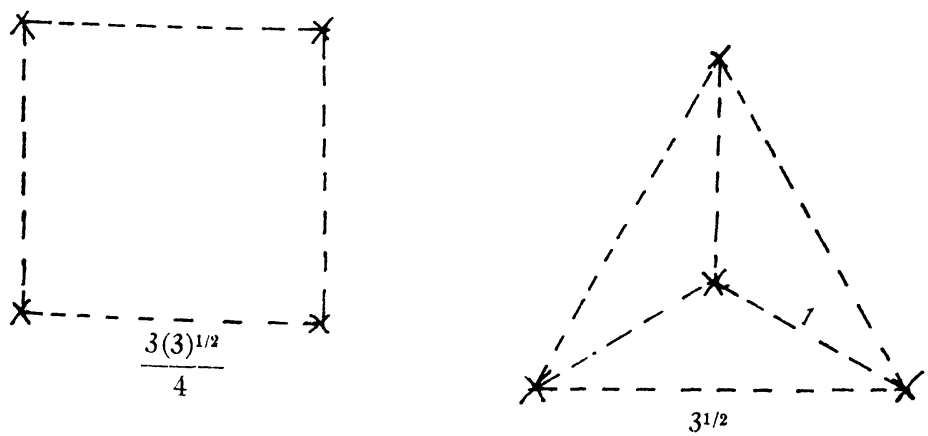

Figure 1

(II) Circumference spanned
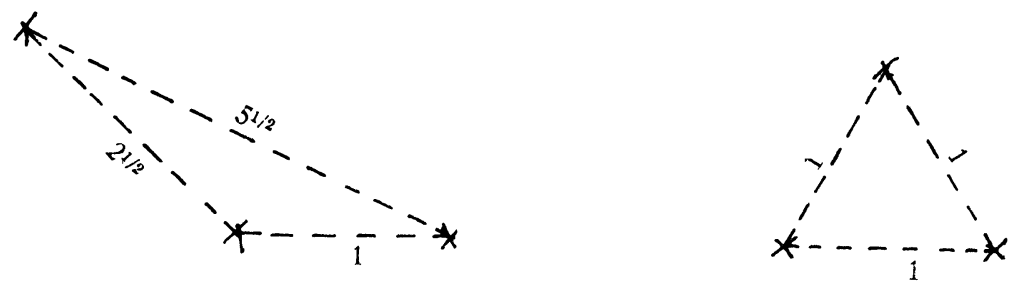

FIGURE 2 
(III) Number of points contributing
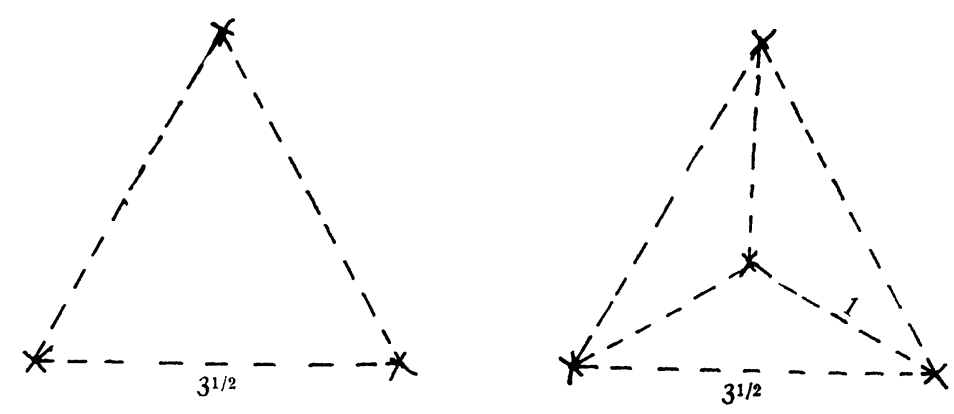

FIGURE 3

Each time the collection of points on the right hand side gives the impression of greater tightness. We are led to introduce for any Minkowski-distance $d$ and for any Jordan polygon $J$ of the affine plane with consecutive vertices $P_{1}, P_{2}, \cdots, P_{n}$ and for any pointset

$$
S=\left\{P_{1}, P_{2}, \cdots, P_{n}, P_{n+1}, \cdots, P_{N}\right\}, \quad N \geqq n,
$$

consisting of the vertices of $J$ and of $N-n$ more points in the interior of $J$ a slackness function of the form:

$$
\sigma(S, J, d)=\frac{A(J)}{k_{2}}+\frac{C(J)}{k_{1}}+k_{0}-N
$$

with suitable positive constants $k_{2}, k_{1}, k_{0}$ and $A(J)$ the area of the pointset $\Pi(J)$ bounded by $J$ and $C(J)$ the Minkowski-circumference

$$
C(J)=\sum_{i=1}^{n} d\left(P_{i}, P_{i+1}\right) \quad\left(P_{n+1}=P_{1}\right)
$$

of $J$. We determine the constants in such a way that the slackness of certain "tightest" configurations becomes zero.

(1) $S$ consists of one point only, resulting in: $k_{0}=1$,

(2) $S$ consists of two points of Minkowski distance 1, resulting in: $k_{1}=2$,

(3) $S$ consists of 3 points of mutual Minkowski-distance 1 forming a basic triangle of a $d$-critical lattice, ${ }^{3}$ leading to the equation:

${ }^{3}$ It can be shown that every $d$-critical lattice contains a basic triangle as indicated. With an arbitrary finite pointset $S$ there is always one corresponding Jordan polygon $J$ associated that has as its vertices some of the points of $S$ such that the remainder of $S$ lies in the interior of $\prod(J)$, viz. the convex hull polygon of $S$. The pointset $\prod(J)$ bounded by $J$ contains any other Jordan polygon that we may come to associate with $S$ in the manner described before. It should be pointed out that we admit a polygon $P_{1} P_{2} \cdots P_{n} P_{1}$ as Jordan polygon as long as it is bounding a pointset such that no two segments intersect in only one point distinct from the 4 vertices involved. Note that $k_{1}$ by its definition has linear dimension. 


$$
\frac{1}{\Gamma^{*}(d) k_{2}}+\frac{3}{2}+1-3=0
$$

resulting in: $k_{2}=\Gamma^{*}(d)$. Thus the slackness function

$$
\sigma(S, J, d)=\Gamma^{*}(d) A(J)+\frac{C(J)}{2}+1-N
$$

is well determined.

It was conjectured by myself [20] and proved by Norman Oler [12] that the slackness of any finite $d$-admissible pointset $S$ is nonnegative. Moreover, it has been shown by $\mathrm{N}$. $\mathrm{Oler}^{4}$ that the slackness vanishes if and only if $d\left(P_{i}, P_{i+1}\right)=1$ for $i=1,2, \cdots, n$ and if $S$ consists of the points in $\Pi(J)$ of a critical lattice. These may be called the tightest $d$-admissible pointsets. The "if" part of Oler's statement about tightest $d$-packings is a special case of the well known equality

$$
A(J)=(I+B / 2-1) \Delta(L)
$$

that holds for any Jordan polygon $J$ with $B$ vertices belonging to a 2-dimensional lattice $L$ with $B$ points on the boundary of $\Pi(J)$, and with $I$ points in the interior of $\Pi(J)$.

Application of Oler's inequality to the intersection of a $d$-admissible pointset with any large square yields a new proof of C. A. Rogers' theorem: $\Gamma^{*}(d)=\Gamma^{* *}(d)$.

Regarding the proof of the inequality the following remarks are made. A broken path $P Q_{1} Q_{2} \cdots Q_{s-1} Q$, all of whose vertices are disjoint excepting perhaps the coincidence of $P$ and $Q$ is called a linkage of the $d$-admissible pointset $S$ if all of the vertices belong to $S$ and if each segment $Q_{i} Q_{i+1}$ is contained in $\Pi(J)$ and if the Minkowski length of each of the segments of the broken path is $1: d\left(Q_{i}, Q_{i+1}\right)$ $=1\left(Q_{0}=P, Q_{s}=Q, i=0,1, \cdots, s-1\right)$. Though the slackness function is not a density function as defined in the beginning of this article it is additive in the sense that:

$$
\sigma(S, J, d)=\sigma\left(S_{1}, J_{1}, d\right)+\sigma\left(S_{2}, J_{2}, d\right)
$$

whenever the Jordan polygon $J$ is cut along a linkage $P_{i} Q_{1} \cdots Q_{s-1} P_{j}$ ( $i \leqq j ; s>0$ or $s=0, n>2, j \neq i-1, j \neq i, j \neq i+1)$ of two vertices $P_{i}$, $P_{j}$ of $J$ via points $Q_{1}, \cdots, Q_{s-1}$ of $S$ in the interior of $\Pi(J)$ such that two new Jordan polygons

\footnotetext{
4 To be published soon. Here the case of $d$ determined by a parallelogram must be excepted.
} 


$$
\begin{aligned}
& J_{1}=P_{i} Q_{1} \cdots Q_{8-1} P_{j} P_{j+1} \cdots P_{n} P_{1} \cdots P_{i}, \\
& J_{2}=P_{j} Q_{s-1} \cdots Q_{1} P_{i} \cdots P_{j}
\end{aligned}
$$

are obtained where the number $N_{i}$ of points of the intersection $S_{i}$ of $S$ with $\Pi\left(J_{i}\right)$ is smaller than $N$.

A permissible variation of a $d$-admissible pointset is defined by means of a continuum $\tau(t)(0 \leqq t \leqq 1)$ of translations of a subset $S_{0}$ of $S$ such that

(1) each point set $\tau(t) S_{0} \cup\left(S-S_{0}\right)$ is $X$-admissible,

(2) $\tau(0)=$ Identity.

Its result is the pointset $\tau(1) S_{0} \cup\left(S-S_{0}\right)$.

If there are points of $S$ in the interior of $\Pi(J)$ then after a sequence of finitely many permissible variations of points of $S$ in the interior of $\Pi(J)$ either all interior points of $\Pi(J)$ which belong to $S$ are moved to the boundary or a linkage between two vertices of $J$ via points of $S$ in the interior of $\Pi(J)$ is obtained. Note that the slackness of the pointset remains the same. Applying double induction and cutting along linkages, the proof of N. Oler's inequality is reduced to the case where every point of $S$ is a vertex of $J$. This case then is settled by a subtle variation process which creates new linkages and thus makes it possible to apply the inductional assumption once again.

For the history of the inequality it is of interest that, seven years before Oler's spectacular demonstration of the inequality

$$
\sigma(S, J, d) \geqq 0
$$

for Minkowski-distances $d$ Norman E. Smith proved (11) for the case of $d$ being equal to the norm distance in 2 dimensions as defined above. A critical lattice of $N_{2}$ had been determined by Hurwitz, and later again by Markoff [7] as the algebraic integer lattice of the algebraic number field generated by $5^{1 / 2}$. Its density is $5^{-1 / 2}$ (see the diagram below showing a fundamental parallelogram of the critical lattice and the region

$$
\left.X=\left\{\left(x_{1}, x_{2}\right)|| x_{1} x_{2} \mid \leqq 1\right\}\right) .
$$

The star associated with $N_{2}$ is remarkable for the high amount of affine symmetry represented by its 2-parametric affine symmetry group consisting of the deformations

$$
x_{i}^{\prime}=\alpha_{i} x_{i} \quad\left(\left|\alpha_{1} \alpha_{2}\right|=1\right) .
$$

All $N_{2}$-critical lattices turn out to be equivalent under the affine symmetry group to Hurwitz's critical lattice. 


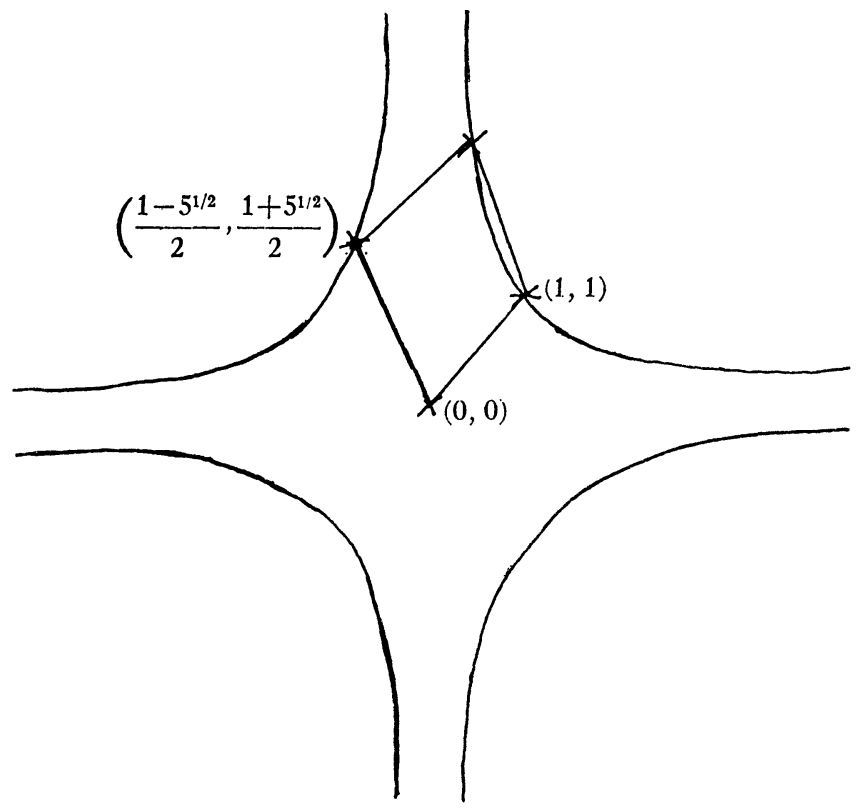

Figure 4

The proof was based on a skillful triangulation of $J$ using the points of $S$ in the interior of $\Pi(J)$ as vertices and on an estimate of the area of the triangles of this triangulation which was derived from the "non-Minkowski" inequality that any $N_{2}$-admissible triangle with side vectors belonging to adjacent quadrants of the $x_{1}, x_{2}$-plane has at least the area $5^{1 / 2} / 2$.

M. Rahman in his thesis of 1957 [13] has further investigated N. E. Smith's method for symmetric 2-dimensional stars with a finite number of asymptotic rays $g_{1}, g_{2}, \cdots, g_{f}$. He found that N. E. Smith's method is only applicable when the distance of a point $P$ of $g_{i}$ from the boundary of the star is of the order of magnitude of the inverse distance of $P$ from $O$ for $i=1,2, \cdots, 2 f$ or possibly of larger order of magnitude, in other words the starshaped regions must be sufficiently blunt near the asymptotes. It was not decided when the inequality obtained by the application of N. E. Smith's method would be sharp.

M. Rahman developed another method solving the packing problem of statistical number geometry for non convex stars $X$ of the $n$-vector space containing $O$ in the interior, namely the domain of action method. It is based on the fact noted by Minkowski that $X$ contains a bounded closed centrally symmetric convex subset $C$ with $O$ in the interior and that consequently the Minkowski distance $d_{C}$ 
associated with $C$ is never smaller than the star distance $d_{x}$ associated with $X$. The $d_{C}$-domain of action of a point $P$ with respect to a pointset $S$ is defined as the set of all points $Q$ satisfying the inequalities

$$
d_{C}(P, Q) \leqq d_{C}\left(P^{\prime}, Q\right) \quad\left(P^{\prime} \neq P, P^{\prime} \in S\right) .^{5}
$$

If $S$ is an $n$-dimensional geometric lattice then the $d_{c}$-domain of action is bounded and measurable, though not necessarily convex, and covariant with the translations of the lattice. Hence the domain of action of any lattice point is equal in volume to the mesh of the lattice.

Generally speaking the minimum volume of the domain of action of the points of a point set $S$ gives an estimate from below of the inverse density of $S$. It is a simple exercise of plane geometry to verify that the domain of action of any point of a plane pointset in which any two points have Euclidean distance not smaller than unity is either infinite or a convex polygon of at most 6 vertices and of an area that is at least equal to $3^{1 / 2} / 2$. Thus another proof of C. A. Rogers' theorem is obtained in the case of the packing problem for circular coins of equal diameter. If one wants to place without overlapping as many coins of diameter equal to unity as possible on a large rectangle the maximum is attained by placing the centers on all those lattice points of some critical lattice for Euclidean distance that lie in the rectangle far enough away from its boundary.

In general, taking $X$ to be a plane star, the aim of the investigation is to obtain a good estimate for the greatest lower bound $k^{* *}$ of the area of the domain of action of a point $P$ with respect to a $d_{X}$-admissible pointset containing $P$. Since $1 / k^{* *} \geqq \Gamma^{* *}$, any estimate of $k^{* *}$ from below leads to an estimate of $\Gamma^{* *}$ from above.

This method has been applied by M. Rahman [13] with good though not sharpest possible results to the hexagonally symmetric star $Y$ that is generated by the $60^{\circ}$-sector

$$
\left\{\left(x_{1}, x_{2}\right)\left|x_{1}^{2}-3 x_{2}^{2} \leqq 1, \quad x_{1} \geqq 3\right| x_{2} \mid\right\} .
$$

A critical lattice of this star was determined by using Bambah's results on stars of hexagonal symmetry [1].

In order to test the strength of the domain of action method in a more decisive manner, it was pitted by Sr. Mary Robert von Wolff [19] against Smith's method in the case of the packing problem of statistical geometry of numbers associated with the norm distance $N_{2}$

- An adjustment must be made if the line $p p^{\prime}$ should happen to be parallel to a straight segment on the boundary of $X$. 
in the plane. She found that for any point $P$ of an $N_{2}$-admissible plane point set $S$, the area of the domain of action of $P$ with respect to $S$ and the Minkowski-distance $d$ determined by

$$
d\left(\left(x_{1}, x_{2}\right),\left(y_{1}, y_{2}\right)\right)=\left(\left|y_{1}-x_{1}\right|+\left|y_{2}-x_{2}\right|\right) / 2
$$

is not smaller than $5^{1 / 2}$. Thus the density of $S$ is not larger than $5^{-1 / 2}$ confirming Smith's result that $\Gamma^{*}\left(N_{2}\right)=\Gamma^{* *}\left(N_{2}\right)$.

The following lemma on the minimum of functions of several real arguments is useful: Let $f, g_{1}, g_{2}, \cdots, g_{t}$ be a finite number of real valued differentiable functions defined on an open subset $D$ of the real $n$-vector space. Let $f$ be restricted to the subset of $D$ that is characterized by the inequalities: $g_{i}(x) \geqq 0(i=1,2, \cdots, t)$. Suppose $f$ has a relative minimum at $x_{0}$ and let, moreover,

$$
\begin{array}{lr}
g_{i}\left(x_{0}\right)=0 & (1 \leqq i \leqq s), \\
g_{i}\left(x_{0}\right)>0 & (i>s) .
\end{array}
$$

Then the $n$-vectors $\nabla f\left(x_{0}\right), \nabla g_{1}\left(x_{0}\right), \cdots, \nabla g_{s}\left(x_{0}\right)$ are linearly dependent. ${ }^{6}$

The results on nonconvex stars mentioned so far, give the impression that C. A. Rogers' result that $\Gamma^{*}-\Gamma^{* *}$ for Minkowski-distances in the plane might go over for all plane stars. Though this might be true for a large class of particularly shaped stars, say with an asymptotic behavior similar to the asymptotic behavior of the $N_{2}$-star, it certainly does not hold for all stars. Sr. Mary Robert von Wolff [19] has discovered a bounded symmetric nonconvex star $X$ of the form (see diagram on page 438 ) for which $\Gamma^{* *}=.5>\Gamma^{*}$. It is highly interesting to observe that one of the densest $X$-admissible point sets turns out to be a "lattice with a base," i.e. a pointset which is the union of a finite number of translates of a geometric lattice of finite nonzero density. ${ }^{7}$ The fact that (vaguely formulated) optimal discrete distributions tend to be lattices with a base has been known to every scientist interested in solid state physics since Bragg's and v. Laue's discoveries.

Is it perhaps possible to reformulate the principle that was vaguely stated above more clearly and precisely in the light of a packing problem of 3-dimensional statistical geometry of numbers? Is it reasonable to assume that lattices with a base form a pattern of optimal packings?

' Otherwise there would be a vector $u$ satisfying $\nabla f\left(x_{0}\right) \cdot u=\nabla g_{1}\left(x_{0}\right) \cdot u=\ldots$ $=\nabla g_{s}\left(x_{0}\right) \cdot u=\epsilon>0$, and, if $\epsilon$ is small enough, $f\left(x_{0}+u\right)<f\left(x_{0}\right), g_{i}\left(x_{0}+u\right)>0(1 \leqq i \leqq t)$, a contradiction.

7 In this case only 2 translates. 
In conclusion speaking more generally of the problems of 3-dimensional statistical number geometry (none of which to my knowledge has been solved), the following problems emerge at once:

(1) What happens if the domain of action method is applied to Euclidean distance where one knows that $\Gamma^{*}=2^{1 / 2}$, or to $N_{3}$ where one knows since Davenport's break through [3] that $\Gamma^{*}=1 / 7$.

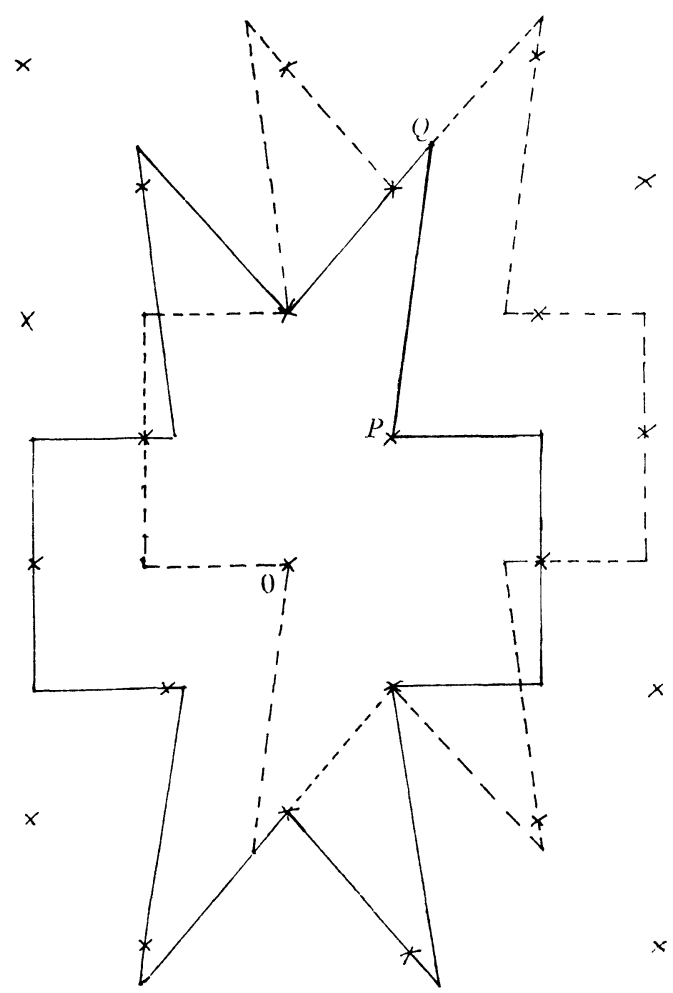

FiguRE 5

$$
\begin{aligned}
& O=(0,0), \\
& P=\left(\frac{7}{16}, \frac{1}{2}\right), \\
& Q=\left(\frac{7}{12}, \frac{5}{3}\right) .
\end{aligned}
$$

(2) Does there exist a slackness function satisfying an inequality similar to the one of $\mathrm{N}$. Oler? 
I would like to thank Dr. L. J. Mordell for his criticism and advice which helped to improve the clarity of the presentation.

\section{BIBLIOGRAPHY}

1. R. P. Bambah, On the geometry of numbers of nonconvex star-regions with hexagonal symmetry, Philos. Trans. Roy. Soc. London. Ser. A vol. 243 (1951) pp. 431-462.

2. J. W. S. Cassels, An introduction to the geometry of numbers, Die Grundlehren der mathematischen Wissenschaften, Vol. 99, Berlin, Springer, 1959.

3. H. Davenport, On the product of three homogeneous linear forms. I-IV, Proc. London Math. Soc. vol. 44 (1938) pp. 412-431; J. London Math. Soc. vol. 16 (1941) pp. 98-101; Proc. Cambridge Philos. Soc. vol. 39 (1943) pp. 1-21. 312.

4. Edmund Hlawka, Zur Geometrie der Zahlen, Math. Z. vol. 49 (1943) pp. 285-

5. L. Fejes Toth, Über die dichteste Zusammenstellung von Kreisen in der Ebene, Christiania Vid.-Selskab Skr., 1910, Nr. 1, 9 pp.

5a. - Some packing and covering theorems, Acta Sci. Math. Szeged vol. 12 (1950) pp. 62-67.

6. K. Mahler, On star-regions in number geometry, Proc. Roy. Soc. London. Ser. A vol. 187 (1946) pp. 151-187.

6a. ——, Nederl. Akad. Wetensch. vol. 49 (1946) pp. 331-343, 444-454, 524$532,622-631$.

7. André Markoff, Sur les formes quadratiques ternaires indéfinis, Math. Ann. vol. 56 (1903) pp. 233-251.

8. H. Minkowski, Diophantische Approximationen, Leipzig, 1907, viii +235 pp.

9. - Geometrie der Zahlen, Leipzig, 1896-1910, 256 pp.

10. L. J. Mordell, On the geometry of numbers in some nonconvex regions, Proc. London Math. Soc. vol. 48 (1945) pp. 339-390.

11. - Geometry of numbers, Proc. First Canadian Math. Congress, Montreal, 1945, Toronto, Univ. of Toronto Press, 1946, pp. 265-284.

12. Norman Oler, On an inequality in number geometry, McGill Ph.D. thesis, 1957; Acta Math. vol. 105 (1961) pp. 19-48.

13. M. Rahman, On some problems of statistical number geometry, McGill Ph.D. thesis, 1957.

14. C. A. Rogers, A note on coverings and packings, J. London Math. Soc. vol. 25 (1950) pp. 327-331.

15. - The closest packing of convex two-dimensional domains, Acta Math. vol. 86 (1951) pp. 309-321, and corrigendum, vol. 104 (1961) pp. 305-306.

16. Carl Ludwig Siegel, $A$ mean value theorem in geometry of numbers, Ann. of Math. vol. 46 (1945) pp. 340-347.

17. Norman E. Smith, On a packing problem of statistical number geometry, Ph.D. thesis, McGill University, 1951.

18. A. Thue, Über die dichteste Zusammenstellung von Kreisen in der Ebene, Christiania Vid.-Selskab Skr., 1910, Nr. 1, 9 pp.

19. Sr. Mary Robert von Wolff, On densest packings for two stars in the plane, Ph.D. thesis, University of Notre Dame, 1961.

20. Hans J. Zassenhaus, Methoden und Probleme der modernen Algebra, Selbstverlag Universitaet Hamburg, 1947.

University of Notre DAME 